\title{
View field selective film of Mono-component polyester composites
}

\author{
Fumihito NOGUCHI, Yasuhiro IWASHIGE, Akiyoshi TAKENO, Minoru MIWA and \\ Teruyuki YOKOI \\ Faculty of Engineering, Gifu University, 1-1 Yanagido, Gifu-shi, Gifu, 501-1193 Japan TEL: \\ x81-058-293-2629 FAX: x81-058-230-1893 e-mail: takeno@apchem.gifu-u.ac.jp \\ (Received 2, October 2001 Accepted 7, December 2001)
}

\begin{abstract}
Mono-component polymer composites are made of materials with the two components of the different high order structures. The mono-component polyester composites consisted of the craze and non-craze regions, which were processed the unique crazing technique. A Characteristic morphology of this crazed composites indicates that a transparent film from a normal viewpoint and an opaque film from a tilted angle viewpoint (view field selectivity) were observed. Mono-component polyester composites are ecomaterials for the separating-free of components and the suitability of a regenerated PET.
\end{abstract}

Keywords : View field selective film, Craze, Necking, Mono-component polymer composites

\section{INTRODUCTION}

Recently, the polymer composites, which are the excellent performance by the compounding of the materials, are applied various industrial productions. However, the separation of component is a serious problem as the scrapping or recycling processing. Authors have presented mono-component polymer composites, which are made of materials with the two components of different high order structures. ${ }^{1-5}$

Characteristic properties are observed on the mono-component polymer composites that consisted of the craze and non-craze regions by the unique crazing technique. This crazed film displayed a transparency film in normal direction to the film surface and an opaque film at the tilt of the viewpoint. View field selectivity that can control the fields of view through the film, because of problems such as interference of sunlight with displays for automobiles and aircraft and protection of privacy in housing. In this paper, we have explored optical properties of the mono-component composites

\section{EXPERIMENTAL \\ II-A. Specimens}

Virgin PET (bottle grade PET), regenerated PET(used PET bottles) and optical PET (O-PET), which were substituted a ethylene glycol unit in a PET structure for 9,9-bis-(4-(2-hydroxyethoy) phenyl)fluorine (BHEPF) as a bulky and rigid main chain and a diol unit with several chain length as a flexible chain, were used. The basic structure of O-PET is shown in Fig. 1. Synthesized O-PET was termed the abbreviation as follows. For example,
B $40 d_{5} 20$ is assigned that B40 refer to BHEPF of 40 $\mathrm{mol}^{\%}$ and $\mathrm{d}_{5} 20$ refer to 1,5 -diol of $20 \mathrm{~mol} \%$ (a subscript is the number of carbon in the diol chain). Films were prepared by the solvent $(1,1,1,3,3,3-$ hexafluoro-2-propanol or chloroform) cast method.

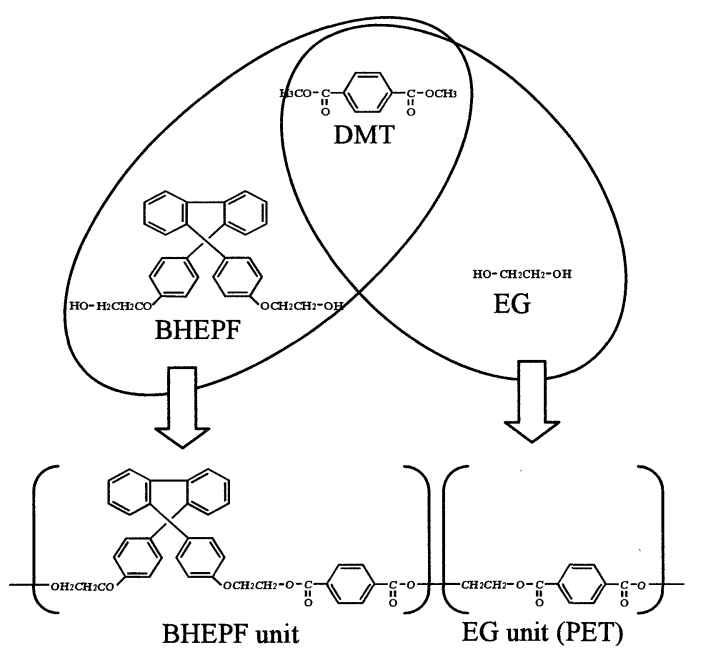

Fig.1. Schematic diagram of O-PET structure.

\section{II-B. Crazing-processing}

The crazing-processing used in this study is shown in Fig. 2, and is essentially the same as that used in a previous paper. ${ }^{6}$ The crazing device was mounted on a tensile tester. The film was bent sharply at the crazing-edge under a constant tension. The pulling tension was controlled by the weight. 
The pulling rate of the crazing-processing is equal to the cross-head speed of the tensile tester and controlled at $10 \mathrm{~mm} / \mathrm{min}$. All experiments were conducted at room temperature.

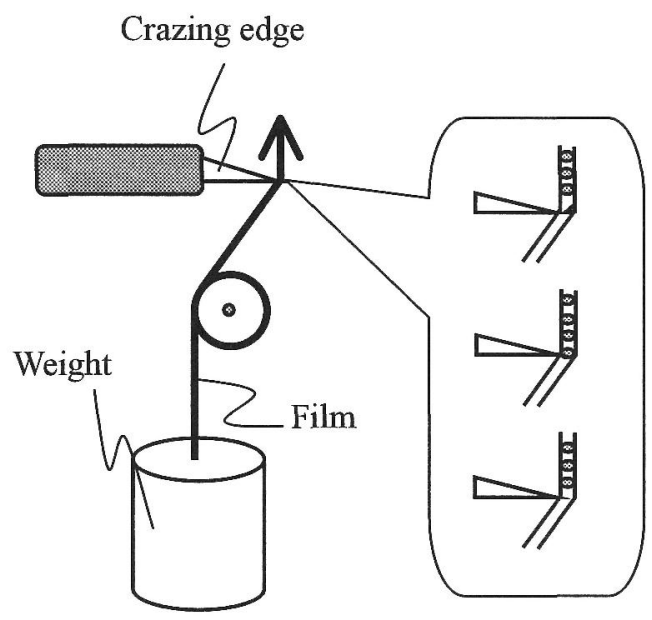

Fig.2. Schematic diagram of crazing processing.

\section{RESULTS AND DISCUSSION \\ III-A. The width and interval of crazes}

In the crazing-processing, the polymer film was bent sharply and the stress was concentrated at the crazing edge as schematically shown in Fig. 2. The craze in the film at the crazing edge is caused by stress without leading to fracture, because the concentrated stress was released at the same time as the generation of the crazes which had the lower Young's modulus and elastic properties as reported for a crazing PVDF in a previous paper. ${ }^{1}$ The crazing edge had been shifted to a non-crazing region of the film according to a moving of the sample film. The stress would have been concentrated and the next craze would have grown in much the same manner as the previous craze, and crazes were caused periodically and continuously.

(1)

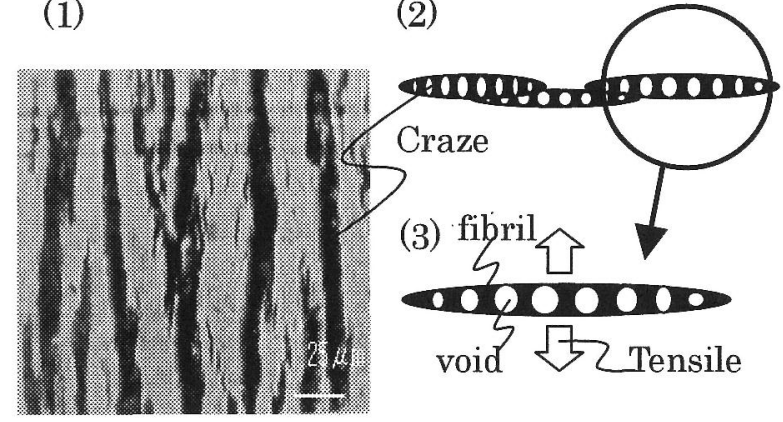

Fig.3. Optical micrograph of crazing film and schematic diagram of craze.
Figure 3-(1) shows the optical transmittance micrographs of the craze composites film. The crazing-processing direction is the horizontal direction in the pictures and crazes are seen as the black stripes perpendicular to the processing direction while the non-craze regions are seen as the white regions. This is because the incident light was scattered by void in the crazes. Crazes were generated uniformly by the crazing processing. Schematic diagram of the craze was shown in Fig. $3-(2)$ and 3-(3). The structure of crazes is composed of fibrils and voids similar to a sponge, however quite different from cracks. Crazes exhibit elastic characteristics while maintaining tensile strength.

The width and interval of the crazes affected optical and mechanical properties of the crazing-processed film. The relationship between virgin PET and regenerated PET were shown in Fig. 4. The width of the crazes increased and the interval (i.e. the non-craze width) decreased with an increase of processing tension. In the other hands, the width of crazes for regenerated PET was larger and the interval was smaller than that of virgin PET. Regenerated PET with a few degradation during the recycle process tended to be generated crazes under mild experimental conditions, because crazing is the precursor phenomenon of polymer fracture.

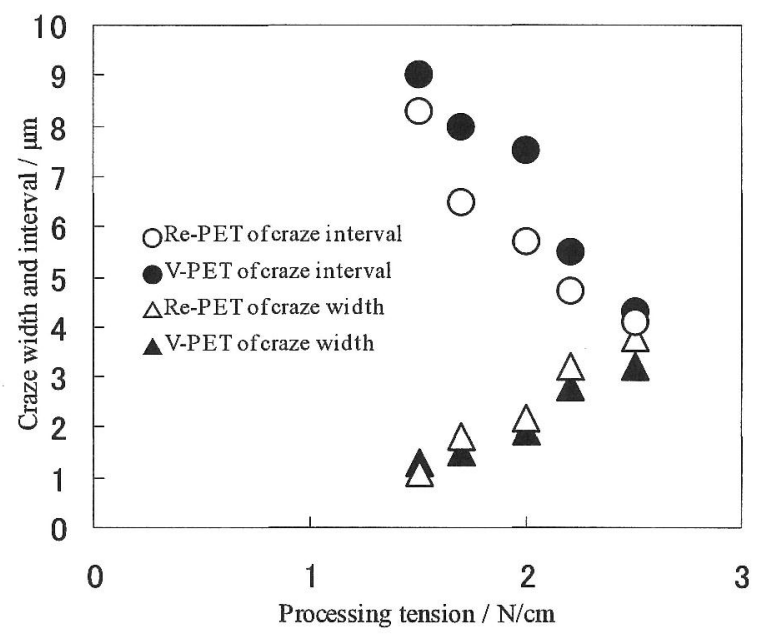

Fig.4. Craze width and interval of PET at various processing tension.

\section{III-B. View field selectivity of PET}

Optical anisotropy of the craze composites film is responsible for the light scattering by the characteristic morphology of the composites. A transparency film in normal direction to the film surface and an opaque film at the tilt of the viewpoint were observed, because the scattering property of incident light depends on the incident 
angle. The craze layers in the film are similar to the slat of a blind. The view field selectivity was estimated by the incident angle dependence of the transmittance using the laser scattering device. Figure 5 and 6 show the result of the incident angle dependence for various processing tension of the virgin and regenerated PET. The processing conditions of favorable view field selectivity are evaluated by the ratio of the transmittance of the narrow angle of incident light and the wide angle of that. In the crazed film of the processing tension of $1.5 \mathrm{~N} / \mathrm{cm}$, typical view field selectivity was observed. As compared with the transmittance at the angle of $0^{\circ}$, the transmittance at the angle of $60^{\circ}$ decreased rapidly with an increase of the processing tension, especially in the regenerated PET film. The transmittance at the incident angle of $0^{\circ}$ depends on the ratio of interval and width of crazes. In the other hand, the transmittance at the wide angle depends on the depth profile of crazes, mainly. As the crazing-processing, it can therefore be presumed that a craze in the regenerated PET grew rapidly to the direction of the film depth by comparison with the craze growth of the virgin PET.

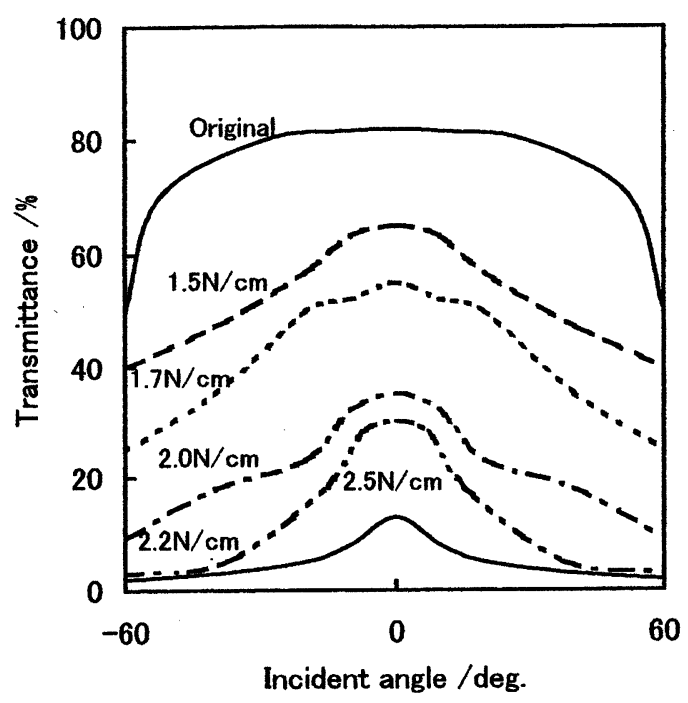

Fig.5. Incident angle dependence of transmittance for virgin PET film at various processing tensions.

\section{III-C. View field selectivity of O-PET}

The growth of crazes depended on the condition of crazing-processing, the molecular structure and the high order structure that is a molecular orientation, crystalline, molecular entanglements and, etc. We synthesized O-PET of various flexibility of the main chain. Optical properties of O-PET were shown in Fig. 7 for examples. Figure 7 indicated that this O-PET was not suitable for view field selectivity and the transmittance decreased with an increase of the processing-tension similar to the virgin PET cases.

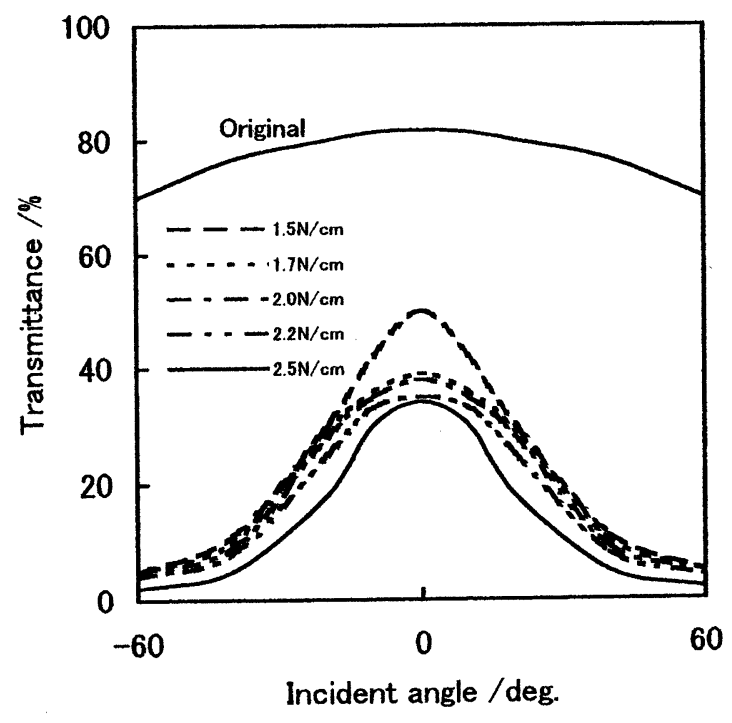

Fig.6. Incident angle dependence of transmittance for regenerated PET film at various pulling tensions.

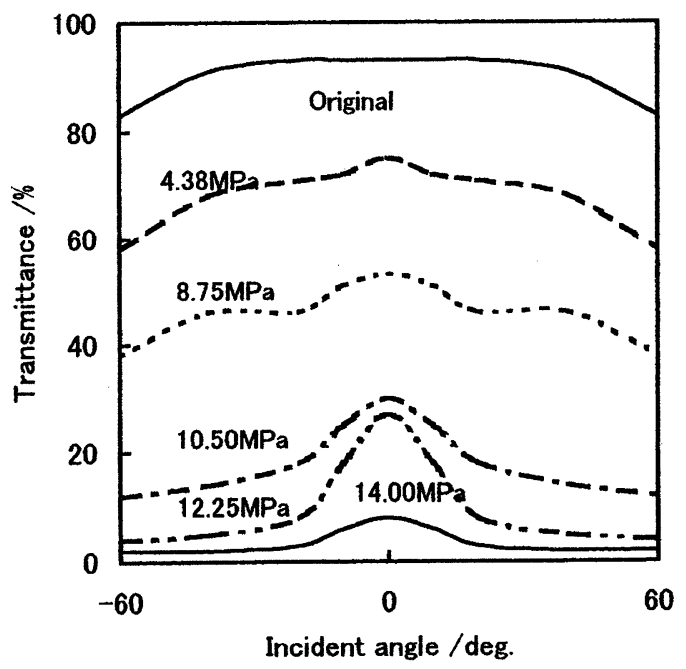

Fig.7. Incident angle dependence of transmittance for O-PET film at various processing tension.

The phase diagram of the molecular structure of O-PET vs. the processing-tension was shown in Fig. 8. The phase diagram is divided into three areas, composites of craze, necking, and both. For O-PET with the long chain length of the diol unit, it was observed that the processing tension allowed for a 
wide range. These O-PET were appropriate correction for the other mono-component composite with a necking region, which can be applied to a lens film for display devices.

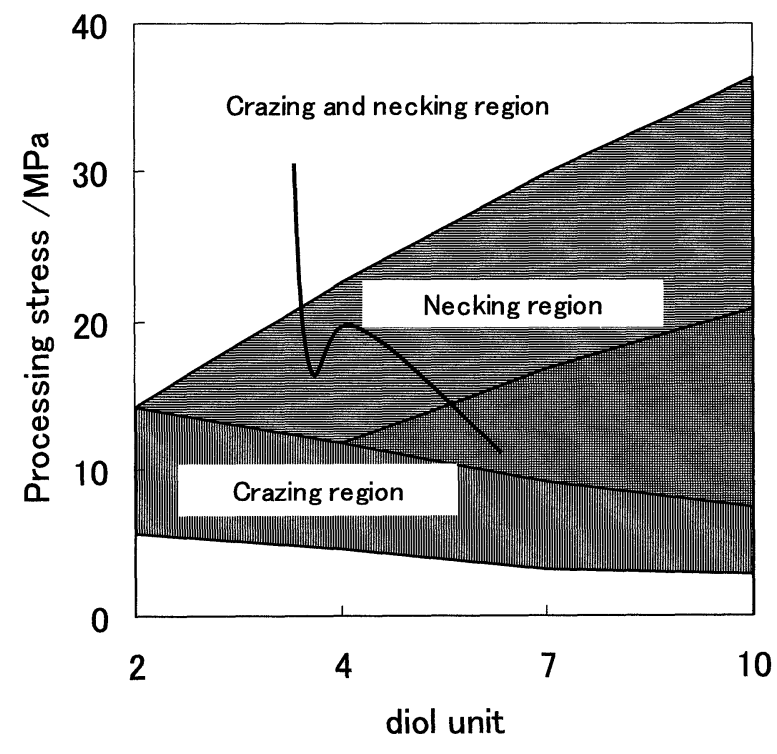

Fig.8. Processing stress of crazing and necking vs. diol unit.

\section{CONCLUSION}

The suitable films for view field selectivity in this study were regenerated PET, virgin PET and O-PET, successively. Advances in the regenerated PET are attributable to anisotropic craze growth in the crazing-processing. The phase diagram of processing tension and the molecular structure from PET to O-PET shows that O-PET films can be optimized for necking composite film.

\section{ACKNOWLEDGEMENTS}

This work was supported by NEDO (New Energy and Industrial Technology Development Organization).

\section{REFERENCES}

${ }^{1}$ A. Takeno, Y. Furuse, M. Miwa and A. Watanabe, Adv. Composite Mater., 4, 129 (1994).

${ }^{2}$ A. Takeno, N. Nakagaki and M. Miwa, Adv.Composite Mater., 7, 35(1998).

${ }^{3}$ Jpn Patent, No.3156058.

${ }^{4}$ U.S. Patent, No.6040941

${ }^{5}$ A. Takeno, M. Yoshimura, M. Miwa, and T. Yokoi, Sen'i Gakkaishi, 57, 11, (2001) in press.

${ }^{6}$ A. Takeno and M. Miwa, Kougyozairyo, 48, 4, 104(2000).

Presented at the $5^{\text {th }}$ International Conference on ECOMATERIALS Oct. 2-4, 2001, Honolulu, Hawaii 